\title{
Fluorescence intensity decay shape analysis microscopy (FIDSAM) for quantitative and sensitive live-cell imaging
}

\author{
Sébastien Peter ${ }^{\mathrm{a}}$, Kirstin Elgass $^{\mathrm{b}}$, Marcus Sackrow ${ }^{\mathrm{b}, \mathrm{c}}$, Katharina Caesar ${ }^{\mathrm{a}}$, Anne-Kathrin Born ${ }^{\mathrm{d}}$, \\ Katharina Maniura ${ }^{\mathrm{d}}$, Klaus Harter ${ }^{\mathrm{a}}$, Alfred J. Meixner ${ }^{\mathrm{b}}$, Frank Schleifenbaum ${ }^{\mathrm{a}, \uparrow}$ \\ ${ }^{a}$ Center for Plant Molecular Biology, Department of Plant Physiology, University of Tübingen, \\ Auf der Morgenstelle 1, 72076 Tübingen, Germany \\ ${ }^{b}$ Institute of Physical and Theoretical Chemistry, University of Tübingen, Auf der Morgenstelle 8, \\ 72076 Tübingen, Germany \\ ${ }^{c}$ current address: PicoQuant GmbH, Rudower Chaussee 29 (IGZ), 12489 Berlin, Germany \\ ${ }^{\mathrm{d}}$ Material-Biology Interactions, Empa, Swiss Federal Laboratories for Materials Testing and \\ Research, Lerchenfeldstrasse 5, 9014 St. Gallen, Switzerland
}

\begin{abstract}
Fluorescence microscopy became an invaluable tool in cell biology in the past 20 years. However, the information that lies in these studies is often corrupted by a cellular fluorescence background known as autofluorescence. Since the unspecific background often overlaps with most commonly used labels in terms of fluorescence spectra and fluorescence lifetime, the use of spectral filters in the emission beampath or timegating in fluorescence lifetime imaging (FLIM) is often no appropriate means for distinction between signal and background. Despite the prevalence of fluorescence techniques only little progress has been reported in techniques that specifically suppress autofluorescence or that clearly discriminate autofluorescence from label fluorescence. Fluorescence intensity decay shape analysis microscopy (FIDSAM) is a novel technique which is based on the image acquisition protocol of FLIM. Whereas FLIM spatially resolved maps the average fluorescence lifetime distribution in a heterogeneous sample such as a cell, FIDSAM enhances the dynamic image contrast by determination of the autofluorescence contribution by comparing the fluorescence decay shape to a reference function. The technique therefore makes use of the key difference between label and autofluorescence, i.e. that for label fluorescence only one emitting species contributes to fluorescence intensity decay curves whereas many different species of minor intensity contribute to autofluorescence. That way, we were able to suppress autofluorescence contributions from chloroplasts in Arabidopsis stoma cells and from cell walls in Arabidopsis hypocotyl cells to background level. Furthermore, we could extend the method to more challenging labels such as the cyan fluorescent protein CFP in human fibroblasts.
\end{abstract}

Keywords: FLIM; FIDSAM; confocal microscopy; fluorescence microscopy; fluorescent proteins; autofluorescence

\section{INTRODUCTION}

In the past years fluorescence microscopy became an invaluable tool for cell biology, mainly due to advances in electronics, optics and fluorescent labels. The triumphal course of autofluorescent proteins (AFPs) such as GFP made it possible to label any desired protein within a living cell ${ }^{1}$. Interactions between different proteins were shown by fluorescence resonance energy transfer (FRET) experiments where the interaction partners are fused to different fluorescent labels which are able to transfer the excitation energy as a function of distance, orientation and nature of the chromophores $^{2}$. Fluorescence lifetime imaging (FLIM) makes it possible to record the spatially resolved fluorescence lifetime of an emitting species and to thereby discriminate different fluorescent species or to determine the local environment of a specific fluorophore ${ }^{3}$.

\footnotetext{
${ }^{\dagger}$ To whom correspondence should be addressed. E-mail frank.schleifenbaum@uni-tuebingen.de
}

Imaging, Manipulation, and Analysis of Biomolecules, Cells, and Tissues VIII, edited by Daniel L. Farkas, Dan V. Nicolau, Robert C. Leif, Proc. of SPIE Vol. 7568, 75681N · @ 2010 SPIE · CCC code: 1605-7422/10/\$18 · doi: 10.1117/12.840644 
However, fluorescence studies in cell biology often suffer from a high background fluorescence, known as autofluorescence, from cellular compounds. This corrupts image contrast and may lead to biological misinterpretation of experimental data ${ }^{4}$. Since autofluorescence spectra are broad and unstructured and very often overlap with label fluorescence, this background can hardly be suppressed by the use of specific bandpass filters. When the fluorescence lifetimes are provided as an additional information (FLIM), label and autofluorescence can be disambiguated in some cases $^{5-6}$. However, for similar lifetime values, which are commonly observed, also this discrimination technique tends to fail ${ }^{4}$.

One way to overcome these restrictions in fluorescence microscopy is the overexpression of fluorescent protein-fused proteins to enhance the signal over the autofluorescence level. However, overexpression implies the risk of affecting the native function of a protein and thereby the generation of artifacts. Accordingly, it is desirable to have a technique which specifically suppresses the autofluorescence and hence allows for imaging of AFP-fused proteins under their native promoters ${ }^{1}$.

Although fluorescence applications are wide-spread in cell biology, only little efforts in contrast enhancement techniques have been reported in the literature. One possibility to enhance the dynamic contrast uses different spectral emission characteristics of label and autofluorescence (spectral unmixing) ${ }^{7}$. Although straight-forward, precise knowledge of the respective emission properties of the emitting species is required. Moreover, since a fluorophore's nanoenvironment may largely affect its emission properties, this behavior is hard to predict because a fluorophore in a cell can be located in different compartments with diverse local $\mathrm{pH}$, ion strength and refractive index ${ }^{3,8}$.

In a recent report, we have presented a novel method to enhance the dynamic contrast in confocal fluorescence microscopy ${ }^{9}$. The technique - Fluorescence Intensity Decay Shape Analysis Microscopy (FIDSAM) - is based on FLIM image acquisition with successive image processing leading to enhanced image contrast. In FLIM, a pulsed laser is used as a light source and a fluorescence intensity decay histogram for fluorescence lifetime determination is recorded for every pixel in the image. In FIDSAM, these histograms are compared to a fluorescence intensity decay reference function. We have recently reported the potential of FIDSAM to suppress the autofluorescence background of A.thaliana hypocotyl cell walls by one order of magnitude ${ }^{9}$. Here, we show that FIDSAM is capable of suppressing autofluorescence background of plant chloroplasts, which is often an issue, particularly when red emitting labels are used; furthermore, we show that FIDSAM is also capable of enhancing the dynamic image contrast when a more challenging label such as CFP is used which is by itself not optimally described by the reference functions used here.

\section{EXPERIMENTAL}

Confocal measurements were carried out with a custom-built confocal laser scanning microscope based on a Zeiss Axiovert 135 TV with FLIM extension (Picoharp 300, Picoquant GmbH, Berlin, Germany) $)^{10}$. Pulsed diode lasers ( $t<300$ ns) from Picoquant ( $\lambda=438 \mathrm{~nm}$ for CFP excitation, $\lambda=473 \mathrm{~nm}$ for EGFP excitation) were used as light sources. Focusing of the illumination light and detection occurred through the same microscope objective (Plan-Neofluar, 100x/1,30 oil, Zeiss). Sample scanning and positioning was controlled by a feedback-controlled sample stage (PI, E710.3CD). Backscattered laser light was blocked by a steep edge filter (LP02-473RU-25, Semrock) or a bandpass filter (FF01$469 / 35$, Semrock) in case of excitation at $473 \mathrm{~nm}$ and $438 \mathrm{~nm}$, respectively. The fluorescence light was focused onto the active area of a spectral integrating avalanche photodiode (APD) (SPCM 200, Perkin Elmer). Data acquisition was controlled by a commercial software package (SymPhoTime, Picoquant GmbH, Berlin, Germany). For recording fluorescence spectra, a motorized flip mirror was inserted into the detection path deflecting the beam onto a grating spectrograph (Spectra Pro 300i, Acton Research) equipped with a thermoelectrically cooled CCD camera (Pixis100B, Princeton Instruments).

Image rendering of raw fluorescence intensity data was performed using the software package WSxM ${ }^{11}$. For image processing by FIDSAM a home-built software package (Cell Imaging Lifetime Analysis, CILA) was used.

Normal dermal human fibroblasts (NHDF) were transfected with reporter gene construct CFP-Vinculin by nucleofection using the NHDF Nucleofector kit (VPD-1001, Amaxa Biosystems, Cologne, Germany) following the manufacturer's protocol. After nucleofection cells were cultured in a humidified incubator at $37^{\circ} \mathrm{C}$ with $5 \% \mathrm{CO}_{2} / 95 \%$ air. Cells were fixed with $4 \%$ paraformaldehyde after $48 \mathrm{~h}$ of cultivation.

Arabidopsis thaliana seedlings were grown and prepared for microcopy as described elsewhere ${ }^{12}$. 


\section{RESULTS}

\subsection{Principle of FIDSAM}

Cell autofluorescence spectra are found to be broad and very unstructured and thereby overlap with commonly used fluorescent proteins ${ }^{9}$. Fig.1 shows an autofluorescence spectrum of human fibroblasts (black line) along with emission spectra of the fluorescent proteins CFP (cyan squares), GFP (green dots) and YFP (orange triangles). The broad autofluorescence spectrum strongly overlaps with the spectra of the mentioned fluorescent proteins. Therefore, autofluorescence always contributes to the recorded intensity in fluorescence microscopy when these proteins are used as a fluorescent label. Hence, the use of appropriate spectral filters is not suitable for clear distinction of label and cell fluorescence.

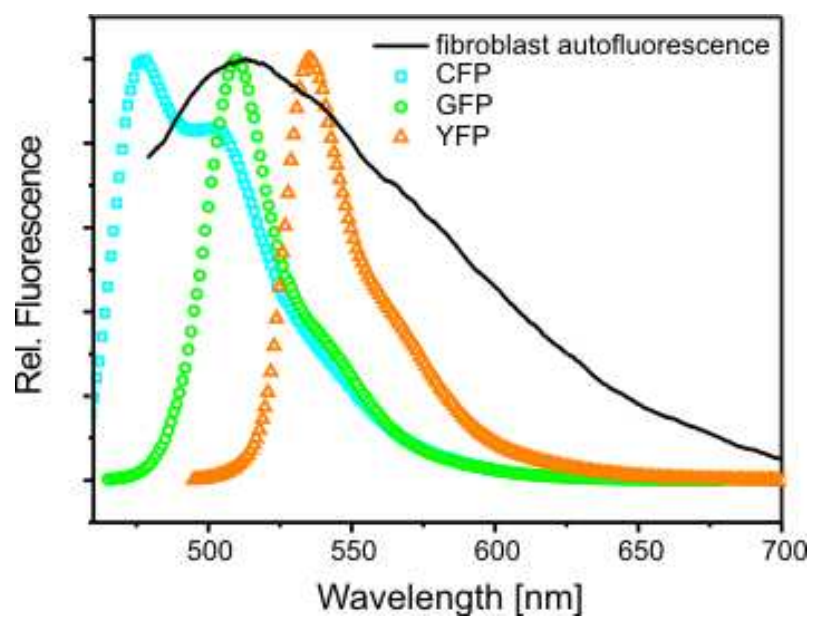

Figure 1 Fluorescence emission spectra of CFP (cyan dots), GFP (green circles) and YFP (orange triangles) along with an autofluorescence spectrum of human fibroblasts.

On the other hand, for the majority of sample systems time gating is no option for a specific suppression of autofluorescence. First, the decay times of autofluorescence and the fluorescent label often are very similar ${ }^{4}$. Second, the fluorescence decay times fluctuate for both label and autofluorescence due to different chemical environments in a complex system such as a cell. As a consequence, a fixed time gating threshold cannot be set.

The key difference between background and label fluorescence lies in the number of different emitters contributing to the respective spectra: Whereas only one emitting species contributes to the label fluorescence, many different emitters contribute to autofluorescence. The temporal evolution of fluorescence obeys first-order kinetics following eq. 1, where $\mathrm{I}(\mathrm{t})$ is the temporal fluorescence intensity, $\mathrm{A}_{\mathrm{i}}$ the contributions of the respective components and $\tau$ the fluorescence lifetime

$$
I(t)=\sum_{i} A_{i} \cdot \exp \left(-\frac{t}{\tau_{i}}\right)
$$

Regarding an ideal fluorescent label, only one component $i$ is required for a satisfying description of the fluorescence intensity decay ${ }^{2}$. Contrarily, many parameters $\mathrm{A}_{\mathrm{i}}$ and $\tau_{\mathrm{i}}$, each representing the contribution of different fluorophores $i$ to the total fluorescence, are needed to describe an autofluorescence intensity decay curve. Fitting a monoexponential decay function (that is $i=1$ ) to experimental FLIM data shows strong deviations for contributing autofluorescence. Hence, the fit quality of a monoexponential reference function is a direct measure for the amount of autofluorescence contributing to a recorded intensity decay curve. For example, EGFP fluorescence is well-fit by a monoexponential decay whereas the decay curve for plant cell wall autofluorescence considerably deviates from a monoexponential ${ }^{9}$. Hence, an increasing contribution of label fluorescence to the total signal leads to a better description of the fluorescence decay curve compared to the pure autofluorescence. We used the error value $\chi^{2}$ which describes the summed deviation of a fit to the normalized experimental data as given in eq. 2 as an objective means for rating the quality of a fit to FLIM data. 


$$
\chi^{2}=\sum_{i} \frac{\left[N\left(t_{i}\right)-N_{c}\left(t_{i}\right)\right]^{2}}{N\left(t_{i}\right)}
$$

Here, $\mathrm{N}_{\mathrm{c}}\left(\mathrm{t}_{\mathrm{i}}\right)$ denotes for the fitted data whereas $\mathrm{N}\left(\mathrm{t}_{\mathrm{i}}\right)$ stands for the experimental data set for every data channel i. For image processing, monoexponential decay curves were convolved with the instrument response function and fit to the normalized spatially resolved FLIM data using a simplex routine. An error image was generated from the pixelwise determined $\chi^{2}$ values using the home-built software CILA. Afterwards, the intensity raw data were sequentially multiplied with the inversed error image which resulted in an enhanced dynamic contrast where autofluorescence contributions are reduced to noise level. For appropriate counting statistics upon fitting lifetime data, several pixels were binned together prior to image contrast correction. For a more detailed description of the FIDSAM technique see?

\subsection{FIDSAM is capable of suppressing chloroplast autofluoroescence}

One prominent example of autofluorescence in fluorescence cell imaging is the bright luminescence of chloroplasts in plants. The far-red emission of chlorophylls can be circumvented by the use of green labels such as (E)GFP and the use of the respective emission filters. Fig.2A shows the fluorescence intensity raw data of Arabidopsis thaliana stoma cells labeled with EGFP at the plasma membrane-associated protein BAK1 under control of its endogeneous promoter. The intensity image reveals brightly fluorescent plasma membranes along with some dim compartments within the stoma cells. These compartments can be identified as chloroplasts when looking at the spatially resolved fluorescence lifetime information (fig.2B). Here, fluorescence lifetime imaging (FLIM) reveals that these inner compartments have a much shorter fluorescence lifetime than the labeled plasma membranes which can then be used for distinction between target signal and autofluorescence. The same information is also revealed via FIDSAM by fitting monoexponential decay functions to the FLIM data and mapping the $\chi^{2}$ values of these fits. Fig.2C shows the error image of these fits with light areas representing regions with the highest $\chi^{2}$ values. The regions identified as chloroplasts on basis of the fluorescence lifetime data coincide with these areas showing major deviations from the fitted reference functions. This reflects the fact that in chloroplasts, housing the light reaction of photosynthesis, complex photophysics occur with kinetics and energy migration processes that are much more complex than a first-order process ${ }^{13}$. Therefore, the analysis of the shape of fluorescence intensity decay curves bears the potential of identifying regions with complex fluorescence kinetics. Finally, one is able to obtain the same information as in FLIM when multiplying the fluorescence intensity raw image with the inversed error image (fig.2D). Using FIDSAM one is capable of selectively suppressing the fluorescence of these regions which follow multiexponential fluorescence intensity decay statistics. In the presented case, the fluorescence intensity of areas which were attributed to chloroplasts on basis of FLIM data are suppressed to background level, opening the way to discriminate between label and autofluorescence on basis of the shape of the fluorescence intensity decay curves. 

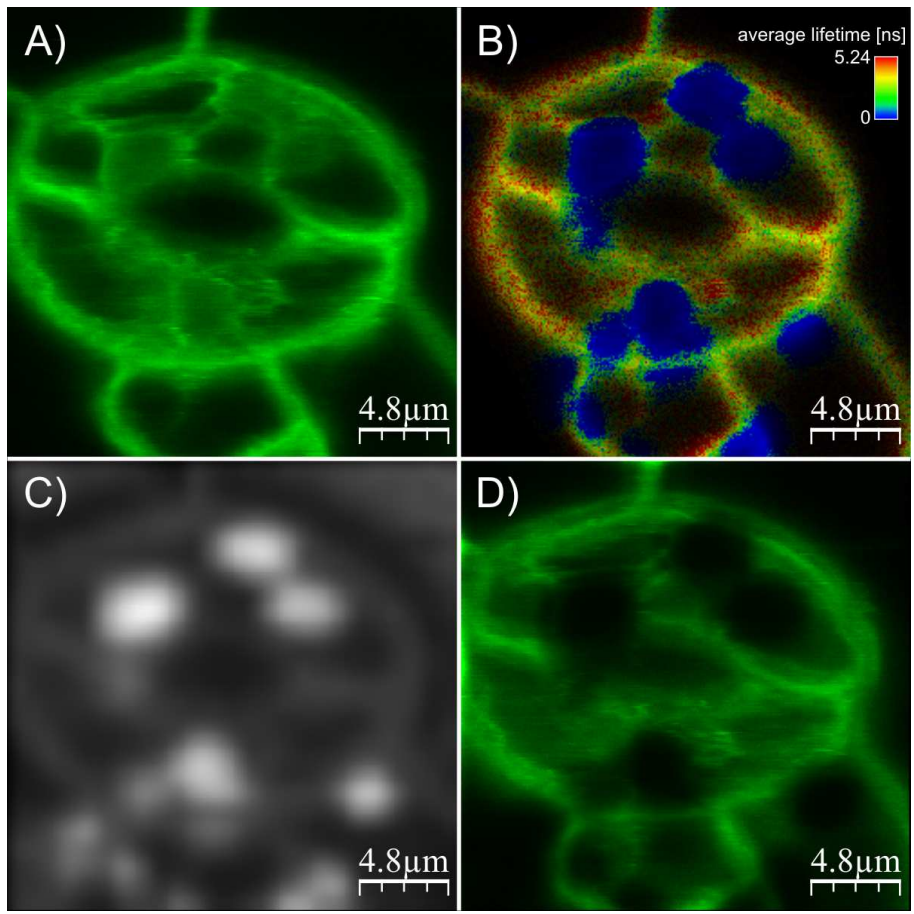

Figure 2 (A) Intensity raw image of Arabidopsis stoma cells labelled with EGFP at the plasma membrane protein BAK1. The chloroplasts are very dim here due to the use of appropriate spectral emission filters. (B) FLIM image of $A$. thaliana stoma cells labelled with EGFP at the plasma membrane-associated protein BAK1. The blue spots represent chloroplasts with fluorescence lifetimes strongly deviating from what is found at the plasma membrane. (C) Error image generated by mapping the spatially resolved deviation of monoexponential decay functions fit to the lifetime data. (D) Corrected intensity image generated by multiplying the raw intensity image with the inversed error image. The regions where chloroplast autofluorescence overlaps with label fluorescence are suppressed to background level.

\subsection{FIDSAM applied to Arabidopsis thaliana plasma membrane-marked hypocotyl cells}

In more challenging cases, autofluorescence overlaps with label fluorescence in terms of both spectral emission and fluorescence lifetime. For example, plant cell walls show a broad fluorescence spectrum similar to the one of fibroblasts shown in fig.1. Furthermore, they show a fluorescence lifetime between 2 and 3 ns which is in the region of some commonly used fluorophores such as EGFP ${ }^{9}$. In these cases, a discrimination between target signal and background cannot be performed on the basis of fluorescence lifetime data.

We imaged hypocotyl cells of 5 days old Arabidopsis thaliana seedlings that expressed the plasma membrane-bound protein LTI6b as an EGFP-fusion ${ }^{14}$. Fig.3A shows an overview image of the recorded EGFP fluorescence. The bright areas show the plasma membrane-bound fluorescence of EGFP and the cell wall autofluorescence which spectrally overlaps with EGFP fluorescence. In a more detailed view (fig.3B) the membranes of adjacent cells cannot be clearly distinguished because the interjacent cell wall fluorescence conceals the low EGFP signal. Spatially resolved fluorescence decay shape analysis with mapping of $\chi^{2}$ values (fig.3C) as described above reveals that the cell wall autofluorescence provides higher error values (gray shaded area in center of fig.3C) than the adjacent plasma membranes. This can then be used for image correction after acquisition by multiplication of the intensity raw data with multiples of the inversed error image. That way, the cell wall autofluorescence can sequentially be suppressed until it reaches background level. Fig.3D shows a FIDSAM image generated by ninefold correction of the intensity raw data with the inversed error image. Areas where mainly autofluorescence contributes to the detected signal have been reduced to background level whereas the regions where mainly EGFP was detected remain largely unaffected; this leads to clear spatial resolution of the plasma membranes of the two adjacent cells. As previously reported, the contrast is enhanced by more than one order of magnitude by application of FIDSAM. This opens the way for quantitative and sensitive fluorescence imaging without the use of overexpressed proteins. 

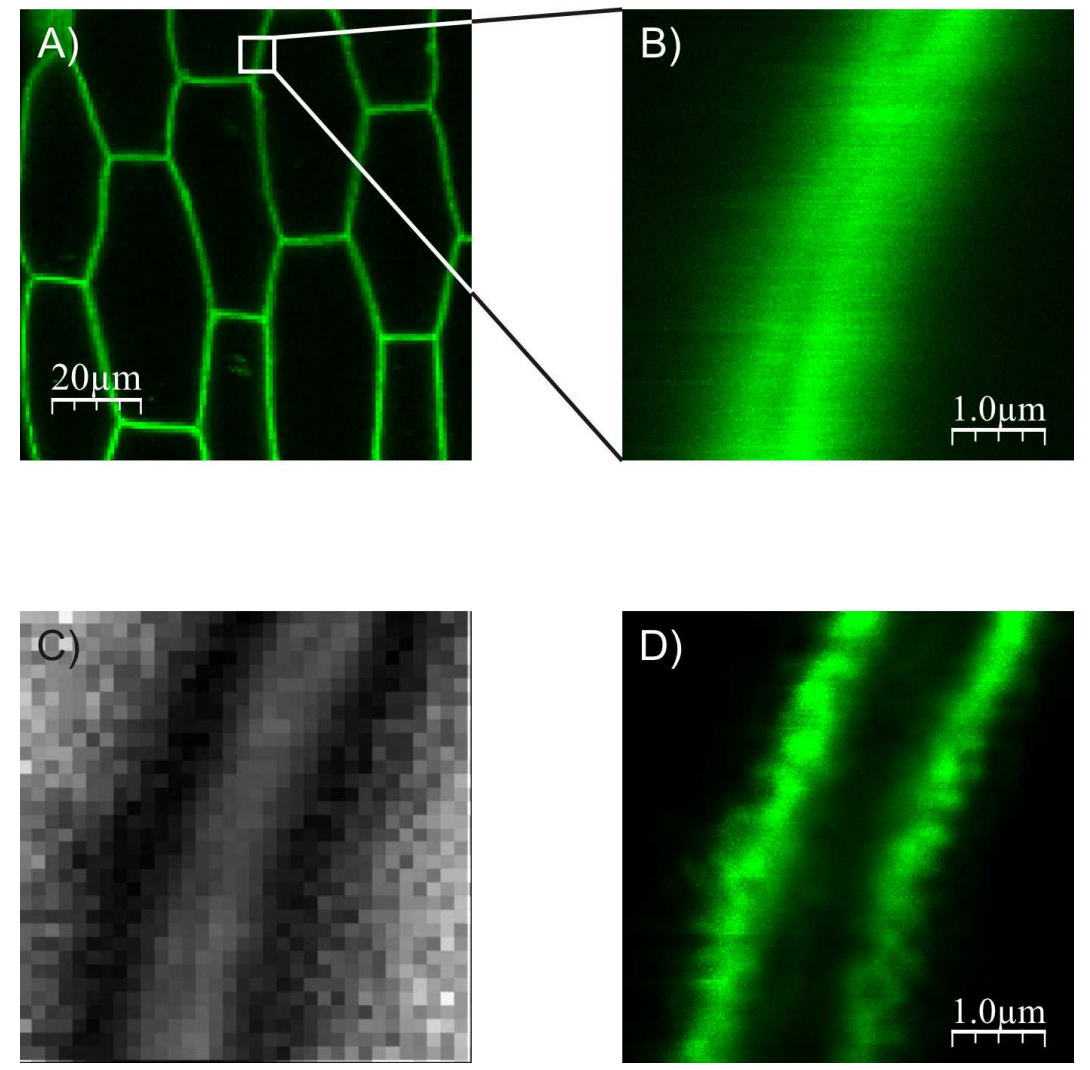

Figure 3 Confocal imaging and contrast enhancement of plant hypocotyl cells labeled at the plasma membrane with EGFP. (A) Overview intensity image. (B) Detailed image of a cell wall. The plasma membranes of adjacent cells are hardly distinguishable due to the cell wall autofluorescence. (C) Error image generated by pixelwise fitting of the FLIM data to monoexponential decay curves and mapping the $\chi^{2}$ values. Bright areas represent regions where the FLIM data show strong deviations from the fitted monoexponential decay curves. (D) Corrected intensity image after ninefold multiplication of the intensity raw-image with the inversed error image. The plasma membranes are clearly distinguishable whereas the cell wall autofluorescence drops to background level.

\subsection{Fluorescent labels with biexponential fluorescence intensity decay statistics: CFP in human fibroblasts}

Up to now, we have shown that FIDSAM is a robust method for discrimination of signal and autofluorescence for EGFP, a label which follows monoexponential fluorescence intensity decay statistics. However, not all fluorescent labels are described by monoexponential decay functions but rather obey a multiexponential decay. Amongst fluorescent proteins, the cyan fluorescent protein CFP is a challenging label for imaging since it provides little brightness, poor photostability and its fluorescence lifetime curve is best fit by a biexponential. Fig.4 shows a monoexponential lifetime fit of EGFP (fig.4A) along with a mono- and biexponential fit of a CFP lifetime curve (fig.4B and C, respectively). Whereas EGFP is best fit by a monoexponential, the CFP lifetime curve is best fit by a biexponential with lifetimes $\tau_{1}=3.5 \mathrm{~ns}$ and $\tau_{2}=1.0 \mathrm{~ns}$, contributing to $90 \%$ and $10 \%$ to the fit, respectively. Monoexponential fits of CFP fluorescence intensity decay curves therefore deviate from experimental data and are always error-prone. The quality of the fit, rated in terms of $\chi^{2}$, is more than one magnitude superior in case of a biexponential fit $\left(\chi^{2}=12\right)$ compared to a monoexponential $\left(\chi^{2}=167\right)$. Although the short lifetime component of CFP only contributes by $10 \%$ to the total fluorescence intensity decay curve, this may impose a severe obstacle to FIDSAM since the critical parameter, $\chi^{2}$, is considerably increased by this minor component. 

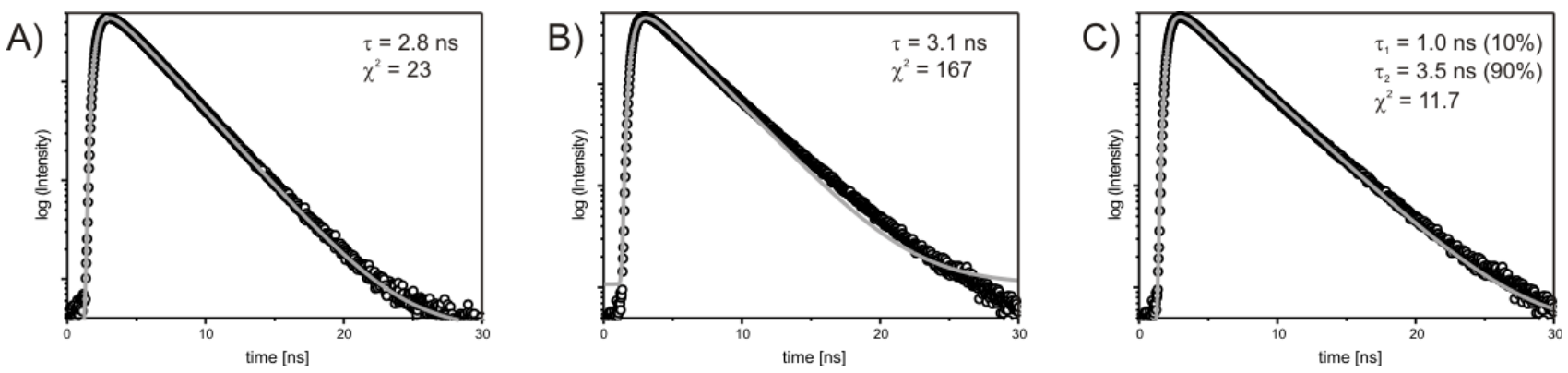

Figure 4 Fluorescence intensity decay histograms (black dots) and lifetime fits (gray lines) of (A) GFP with a monoexponential fit; (B) CFP with a monoexponential fit; (C) CFP with a biexponential fit. Whereas the GFP fluorescence intensity decay histogram is fairly fit by a monoexponential, the quality of the fit to the CFP lifetime curve substantively increases when fitting a biexponential instead of of monoexponential, emphasized by a decrease of the $\chi^{2}$ value by more than one order of magnitude. Data were fit using the program IBH decay analysis software (Horiba Jovin Yvon).

For demonstrating that FIDSAM also works under these more stringent conditions without any changes in the image correction process, we have performed measurements with human fibroblasts overexpressing vinculin as a CFP fusion protein. Vinculin is located in focal adhesion sites and plays a role in linking integrins to actin. Confocal imaging of these fibroblasts reveals elongated plaques and a large number of spherical compartments that strongly fluoresce (fig.5a). Monoexponential fits of the fluorescence intensity decay curves and mapping the error values $\chi^{2}$ shows that the longish plaques, typical for focal adhesion sites, show satisfyingly small deviations from the applied monoexponential reference functions whereas the fluorescence intensity decay curves of the spherical compartments are more error-prone (fig.5B). Fig.5C and D show FIDSAM images where the original intensity image has been corrected with the inversed error image four and seven times, respectively. These corrected images reveal that the focal adhesion sites remain largely unaffected whereas the spherical compartments gradually vanish.

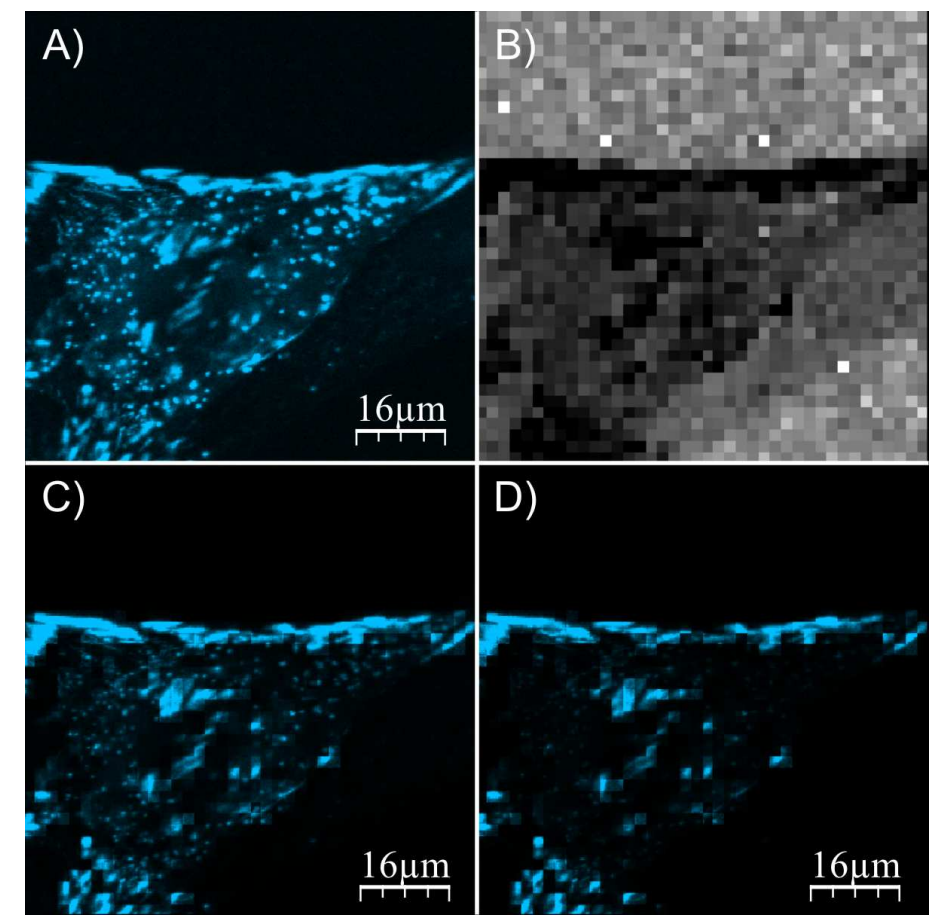

Figure 5 (A) Intensity image of a human fibroblast overexpressing CFP-Vinculin. (B) Error image calculated by fitting monoexponential decay functions to the respective FLIM data. (C) and (D) Corrected intensity images generated by fourfold and sevenfold multiplication of the intensity raw image with the inversed error image. Mainly the focal adhesion sites remain visible whereas autofluorescent vesicles are suppressed to background level. 
This is an interesting finding since CFP lifetime fits intrinsically provide high $\chi^{2}$ values when applying FIDSAM. We attribute the finding that CFP fluorescence is less affected by the correction procedure than autofluorescence to the fact that for FIDSAM, the relative fit quality is rather critical than the absolute one. The data shown here are therefore explained if one takes into consideration that FIDSAM provides contrast enhancement as long as the used label is better described by the mentioned fitting algorithm than background fluorescence. Therefore, CFP lifetime curves, although error-prone, are still better described by a monoexponential decay function than those of autofluorescence. The fact that CFP is less suppressed by FIDSAM than autofluorescence emphasizes the robustness of the applied method and opens the way to broader applicability with challenging labels.

\section{SUMMARY AND CONCLUSION}

In this report, we have shown that a novel method in fluorescence microscopy, FIDSAM, enables one to significantly increase the dynamic contrast by reduction of the autofluorescence background. The method makes use of the different shape of fluorescence lifetime curves of background and label fluorescence. The key difference between the two types of fluorescence lies in the number of contributions necessary for analytically describing the respective fluorescence lifetime curves. Starting from samples where target and autofluorescence can be discriminated on basis of their fluorescence lifetimes we could show that FIDSAM provides a way to disambiguate a fluorescent label in an autofluorescent environment without the generation of artifacts. That way, we were able to show that autofluorescence lifetime curves originating from plant chloroplasts significantly differ from the lifetime curve of the fluorescent protein EGFP. When applied to EGFP-labeled plasma membranes in plant cells, we showed that the plasma mebranes of two adjacent cells, which cannot be separated by state-of-the-art confocal microscopy, can be spatially resolved by correcting the original image with FIDSAM. Finally, we could show that the contrast enhancement method can also be extended to labels where there is more than one single component contributing to the fluorescence intensity decay curve. These results show the robustness and the broad range of applications of FIDSAM and open the way to quantitative fluorescence imaging of low-expressed proteins without background interference.

\section{ACKNOWLEDGEMENT}

This work was funded by grants from the State of Baden-Württemberg and the University of Tübingen to S.P., F.S., K.E. and K.C., and a DFG grant to K.H. (HA2146/10-1).

\section{REFERENCES}

[1] Y. Wang, J. Y.-J. Shyy, and S. Chien, "Fluorescence Proteins, Live-Cell Imaging, and Mechanobiology: Seeing Is Believing," Annual Review of Biomedical Engineering, 10 (1), 1-38 (2008).

[2] J. R. Lakowicz, [Principles of Fluorescence Spectroscopy] Kluwer Academic, New York(1999).

[3] T. Nakabayashi, H.-P. Wang, M. Kinjo et al., "Application of fluorescence lifetime imaging of enhanced green fluorescent protein to intracellular pH measurements," Photochem. Photobiol. Sci., 7 668-670 (2008).

[4] N. Billinton, and A. W. Knight, "Seeing the wood through the trees: a review of techniques for distinguishing green fluorescent protein from endogenous autofluorescence," Anal Biochem, 291 (12), 175-97 (2001).

[5] A. Esposito, and F. S. Wouters, "Fluorescence lifetime imaging microscopy," Current Protocols in Cell Biology, 4 (14), (2004).

[6] E. B. v. Munster, and T. W. Gadella, "Fluorescence lifetime imaging microscopy (FLIM)" Adv Biochem Eng Biotechnol., 95 143-175 (2005).

[7] M. E. Dickinson, G. Bearman, S. Tille et al., "Multi-Spectral Imaging and Linear Unmixing Add a Whole New Dimension to Laser Scanning Fluorescence Microscopy," BioTechniques, 31 (6), 1272-1278 (2001).

[8] H.-J. v. Manen, P. Verkuijlen, P. Wittendorp et al., "Refractive Index Sensing of Green Fluorescent Proteins in Living Cells Using Fluorescence Lifetime Imaging Microscopy,” Biophys J.: Biophys. Lett., L67-L69 (2008).

[9] F. Schleifenbaum, K. Elgass, M. Sackrow et al., "Fluorescence intensity decay shape analysis microscopy (FIDSAM) for quantitative and sensitive live-cell imaging," Mol. Plant, 10.1093/mp/ssp110 (2009). 
[10] F. Schleifenbaum, C. Blum, V. Subramaniam et al., "Single Molecule Spectral Dynamics at Room Temperature," Mol. Physics., 107 (18), 1923 - 1942 (2009).

[11] I. Horcas, R. Fernandez, J. M. Gomez-Rodriguez et al., "WSXM: A software for scanning probe microscopy and a tool for nanotechnology,” Rev. Sci. Instrum., 78 (1), 013705 (2007).

[12] K. Elgass, K. Caesar, F. Schleifenbaum et al., "Novel application of fluorescence lifetime and fluorescence microscopy enables quantitative access to subcellular dynamics in plant cells," PLoS One, 4 (5), e5716 (2009).

[13] S. Eberhard, G. Finazzi, and F.-A. Wollman, "The Dynamics of Photosynthesis," Annual Review of Genetics, 42 (1), 463-515 (2008).

[14] S. R. Cutler, D. W. Ehrhard, J. S. Griffitts et al., "Random GFP:cDNA fusions enable visualization of subcellular structures in cells of Arabidopsis at high frequency," Proc. Nat. Am. Soc., 97 3718-3723 (2000). 